Musées, Patrimoine et Culture scientifiques et techniques

$118 \mid 2008$

juillet - août 2008

\title{
Visite + : innover dans l'interactivité
}

\section{Roland Topalian et Joëlle Le Marec}

URL : http://journals.openedition.org/ocim/342

DOI : 10.4000/ocim.342

ISSN : 2108-646X

\section{Éditeur}

OCIM

Édition imprimée

Date de publication : 1 juillet 2008

Pagination : 22-32

ISSN : 0994-1908

Référence électronique

Roland Topalian et Joëlle Le Marec, «Visite + : innover dans l'interactivité », La Lettre de I'OCIM [En

ligne], 118|2008, mis en ligne le 01 décembre 2010, consulté le 19 avril 2019. URL : http://

journals.openedition.org/ocim/342 ; DOI : 10.4000/ocim.342 


\section{Visite+ : innover dans l'interactivité}

Roland Topalian et Joëlle Le Marec *

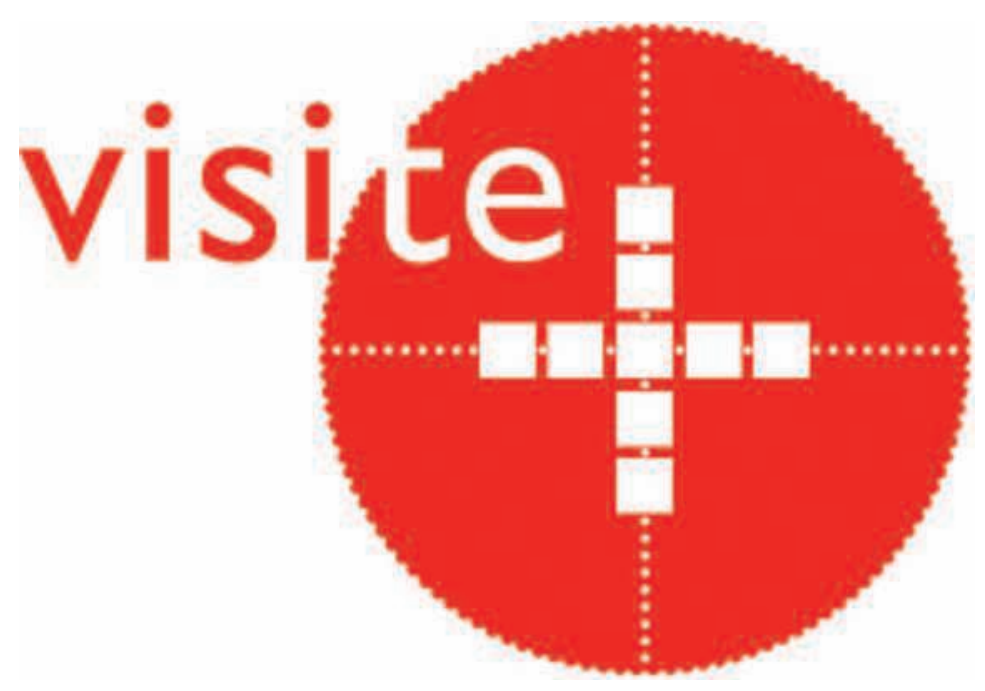

(c) CSI
Innovation culturelle et technologique, le concept Visite+ a comme point de départ la prise en compte des pratiques culturelles des visiteurs. Les initiateurs de l'expérience montrent comment cet outil crée pour les visiteurs un lien nouveau avec le musée au-delà de la simple visite et suscite, dans le cadre d'une recherche-développement collective, une remise en question des formats classique de collaboration entre une institution muséale et un laboratoire de recherche.

2001, les visiteurs de la Cité des Sciences et de l'Industrie découvrent le premier service personnalisé proposé par Visite+, La lettre du visiteur. Il s'agit d'une lettre mensuelle qui propose une information sur les nouvelles activités de la Cité, et, surtout, une veille thématique sur l'activité culturelle à l'échelle nationale autour de thèmes que les visiteurs choisissent. 2002, Le cerveau intime est la première exposition équipée de Visite+. L'expérience est renouvelée dans les expositions La population mondiale et moi puis Changer d'ère. Aujourd'hui Visite+ a été utilisé dans neuf expositions avec à chaque fois une mise en œuvre différente mais un principe général identique : permettre au visiteur de faire une visite en intégrant des éléments personnels (se situer dans les données démographiques, objectiver son empreinte écologique), garder trace de son parcours et de sa visite, et avoir la possibilité de poursuivre chez lui la visite. 
Visite+ n'est pas d'un « outil », un objet technique à propos duquel on pourrait parler d'usages spécifiques (au sens où on l'entendrait pour l'usage d'un téléphone, d'un audio guide, et même d'un site Internet). Pourtant, sa conception s'est fondée sur une prise en compte très forte des pratiques des visiteurs de la Cité des Sciences et de l'Industrie.

Pour le visiteur, il se présente d'abord sous forme d'un code barres sur son ticket d'entrée, ou d'une étiquette électronique figurant sur une carte prise à l'entrée de l'exposition, ou bien sous forme de tout autre objet qui fera office d'identifiant, une fois présenté devant un capteur ad hoc placé dans l'exposition (cimaise, multimédias...). Dans l'exposition, Visite+ se fait discret et se fond dans l'ensemble des activités pratiquées. De retour chez soi, une fois qu'on a saisi sur Internet son code d'accès sur le portail de Visite+, s'ouvre le Cyber-carnet. Le visiteur retrouve alors sur un site personnel des éléments de sa visite et peut continuer à lire, jouer, consulter, approfondir, intervenir à distance sur l'exposition, partager ses opinions avec les autres visiteurs de l'exposition et communiquer à propos de l'exposition ou de sa visite. Par rapport à ses pratiques muséales, Visite+ concrétise un lien à l'institution au-delà du moment de la visite et ouvre la possibilité d'une mémoire personnelle et cumulative de ses visites d'expositions et des pratiques culturelles qui leur sont liées (lecture...).

Pour le concepteur, Visite+ est autre chose : un agencement composite d'outils de communications, évolutif, qui mobilise un ensemble de supports dans plusieurs espaces (au musée, chez soi). Il y a bien création technologique. Mais cette création ne vise pas à la production d'un objet technique achevé. Elle consiste plutôt en un développement " incrémental » qui s'enrichit régulièrement et vit au rythme des besoins qui

\begin{tabular}{|c|c|c|c|}
\hline Expositions & Années & Identifiants & $\begin{array}{l}\text { Nombre de points } \\
\text { de lecture } \\
\text { de l'identifiant }\end{array}$ \\
\hline $\begin{array}{l}\text { Le cerveau } \\
\text { intime }\end{array}$ & 2002 & $\begin{array}{l}\text { Code barre sur } \\
\text { ticket billetterie }\end{array}$ & 27 \\
\hline $\begin{array}{l}\text { Canada } \\
\text { vraiment }\end{array}$ & 2003 & $\begin{array}{l}\text { PDA distribué au } \\
\text { visiteur, une carte } \\
\text { comportant le numéro } \\
\text { de post visite est donnée } \\
\text { lors du dépôt PDA }\end{array}$ & 13 \\
\hline $\begin{array}{l}\text { Opération } \\
\text { carbone }\end{array}$ & 2004 & $\begin{array}{l}\text { Code barre sur } \\
\text { sur ticket billetterie }\end{array}$ & 2 \\
\hline $\begin{array}{l}\text { La population } \\
\text { mondiale } \\
\text { et moi }\end{array}$ & 2005 & $\begin{array}{l}\text { Code barre sur } \\
\text { code spécifique }\end{array}$ & 10 \\
\hline $\begin{array}{l}\text { L'ombre } \\
\text { expliquée } \\
\text { aux enfants }\end{array}$ & 2005 & $\begin{array}{l}\text { Feuille imprimée } \\
\text { à l'unité avec la } \\
\text { photographie imprimée } \\
\text { et numéro de post visite }\end{array}$ & 1 \\
\hline Biométrie & 2005 & $\begin{array}{l}\text { Feuille imprimée } \\
\text { à l'unité avec numéro } \\
\text { de post visite }\end{array}$ & 4 \\
\hline Changer d'ère & 2006 & $\begin{array}{l}\text { Code barre sur carte } \\
\text { spécifique }\end{array}$ & 11 \\
\hline $\begin{array}{l}\text { Science } \\
\text { actualités } \\
\text { opinion } \\
\text { publique }\end{array}$ & 2007 & $\begin{array}{l}\text { Code barre sur carte } \\
\text { spécifique }\end{array}$ & 2 \\
\hline $\begin{array}{l}\text { Cité } \\
\text { des enfants }\end{array}$ & 2007 & $\begin{array}{l}\text { Ticket imprimé } \\
\text { à l'unité avec } \\
\text { photographie imprimée } \\
\text { et numéro de post visite }\end{array}$ & 1 \\
\hline
\end{tabular}
s'expriment pour chaque nouvelle exposition. En effet, dans la mesure où Visite+ est né en réponse à par le dispositif Visite+ ce qui s'exprimait dans les pratiques des visiteurs, il est destiné à évoluer et à "suivre » les visiteurs dont les pratiques culturelles ne sont pas cloisonnées par les frontières entre établissements, et à entrer dans la réflexion muséologique des concepteurs d'expositions (1). 
Enfin, pour le chercheur, Visite+ est une réponse à des résultats d'études des pratiques des visiteurs de la Cité des Sciences et de l'Industrie. Après sa création, il a à nouveau donné lieu à des études dans les expositions (Le cerveau intime, La population mondiale et moi), et à de multiples moments de dialogue, et à des réflexions approfondies sur la personnalisation et sur le lien à l'institution, dans le cadre de conventions reconduites d'année en année entre la Cité des Sciences et de l'Industrie et le laboratoire Communication, Culture et Société de l'École Normale Supérieure Lettres et Sciences Humaines de Lyon (2). Les études menées pour Visite+ n'entrent pas dans le format canonique des traditionnelles études recherche-développement, c'est-à-dire avec d'une part un volet théorique qui s'inscrirait dans la production propre d'un laboratoire et qui nourrirait la publication académique, et d'autre part un ensemble de résultats qui serviraient la collaboration proprement dite. La manière dont les études inspirent le développement, et la façon dont ce développement inspire à son tour les enquêtes, deviennent inextricablement liées.

C'est pourquoi Visite+ suscite actuellement une remise en question des formats classiques de la collaboration entre recherche et développement et suppose une créativité institutionnelle.

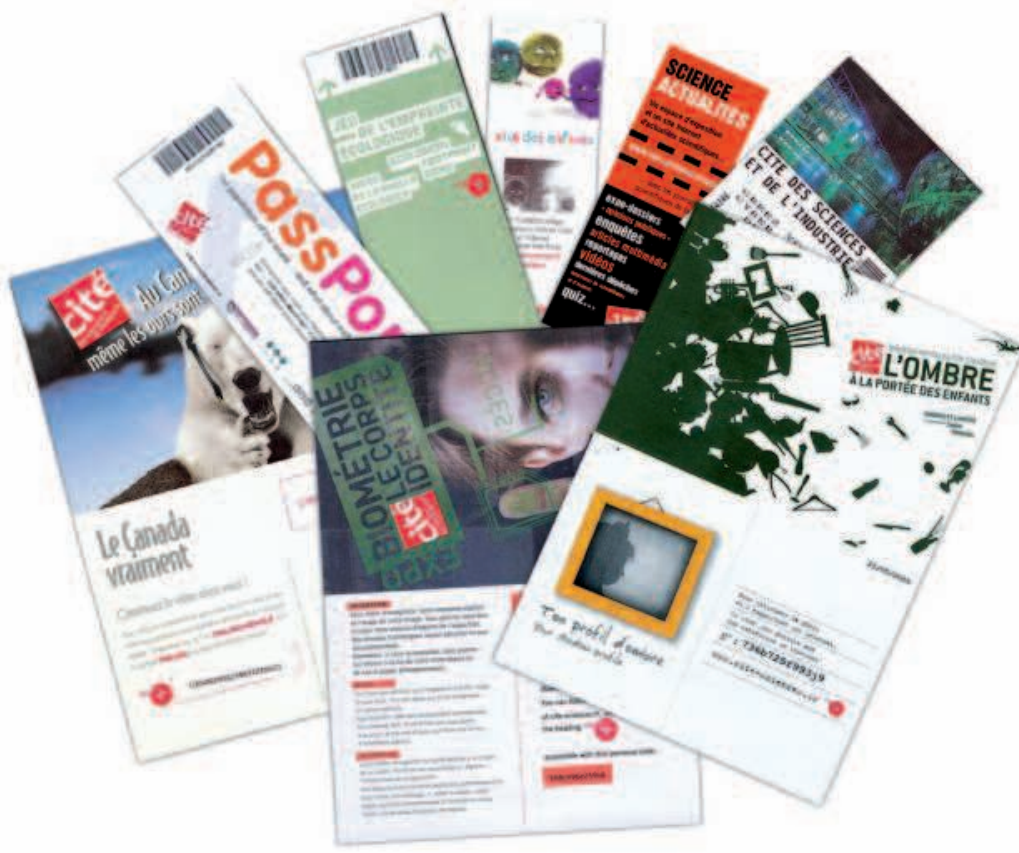

Exemples de supports d'identifiants pour différentes expositions
Dans l'article qui suit, nous allons développer ces quelques points, pour aboutir à une proposition d'extension de Visite+ à un ensemble d'institutions culturelles, dans un processus de recherche-développement et d'innovation institutionnelle conduit collectivement et sur le long terme par des établissements culturels et des laboratoires.

En effet, si Visite+ est une innovation culturelle et technologique, conçue initialement par la Cité des Sciences et de l'Industrie et un laboratoire de recherche, sa logique de développement, du point de vue des pratiques culturelles auxquelles il répond et du point de vue du type de recherche-action dont il est issu, est un processus de création et d'adaptation continu, conduit ensemble par des musées ou structures culturelles, et des laboratoires qui s'associent pour l'exploiter et le faire évoluer. C'est pourquoi le dispositif est proposé en licence gratuite. La Cité des Sciences et de l'Industrie et le laboratoire Communication, Culture et Société de l'ENS Lettres et Sciences Humaines (Lyon) souhaitent ainsi prolonger cette démarche avec les institutions culturelles et laboratoires intéressés dans le cadre d'un groupement «Musée/Recherche » destiné à partager les expériences, les développements et la recherche dans les domaines contenus dans la proposition Visite+ : personnalisation, articulation des temps de la visite, extension de la visite à l'espace Internet.

\section{Le laboratoire de l'interactivité}

Visite+ a été élaboré par le département Interactivité et Multimédia (aujourd'hui DAMI piloté par Isabelle Goutte). Cette structure fondée en 1982 (« La cellule des petits logiciels » par Daniel de Meester) a assuré la conception de l'ensemble des éléments interactifs de la Cité des Sciences. Ce département s’appuie sur une pratique de conception de scénarios élaborés et mis en œuvre au cas par cas. Chaque projet de communication pour chaque contenu scientifique, pour chaque exposition, avec chaque équipe ou conseiller scientifique, dans chaque configuration avec ses contraintes et ses potentialités, est une expérimentation qui enrichit une réflexion générale du département sur la médiation. À cette fin, les moyens technologiques sont souvent détournés de leur fonction d'origine et l'innovation consiste ici en un réagencement, un « tricotage » de ces technologies par des professionnels (ingénieurs, concepteurs, informaticiens) du département Interactivité et Multimédia, au service de projets de médiation, dans le cadre des expositions. La conception des scénarios de médiation interactifs s'appuie donc sur des moyens technologiques ad 
hoc, ou détournés. Aussi, du fait de la nature très particulière de la communication interactive, la conception des scénarios plaçait naturellement le visiteur au centre du projet de communication (3).

Dans une phase initiale (1984-1989) les projets de médiation s'appuyaient sur l'idée d'un utilisateurmodèle, dans une représentation qui empruntait à deux traditions très différentes : la technologie et la didactique. Le visiteur était imaginé comme un utilisateur aux prises avec un outil technique, et comme un apprenant engagé dans une démarche cognitive. Les évaluations ont fait voler en éclat cette représentation de l'usager-apprenant et ont fait exister, au stade de la conception, des représentations bien différentes de nos visiteurs, à la fois plus simples et plus complexes, plus émouvantes, plus proches de nous. Ces résultats, que nous évoquerons plus loin très brièvement ont contribué à transformer la manière dont nous avons travaillé. En dix ans, nous avons

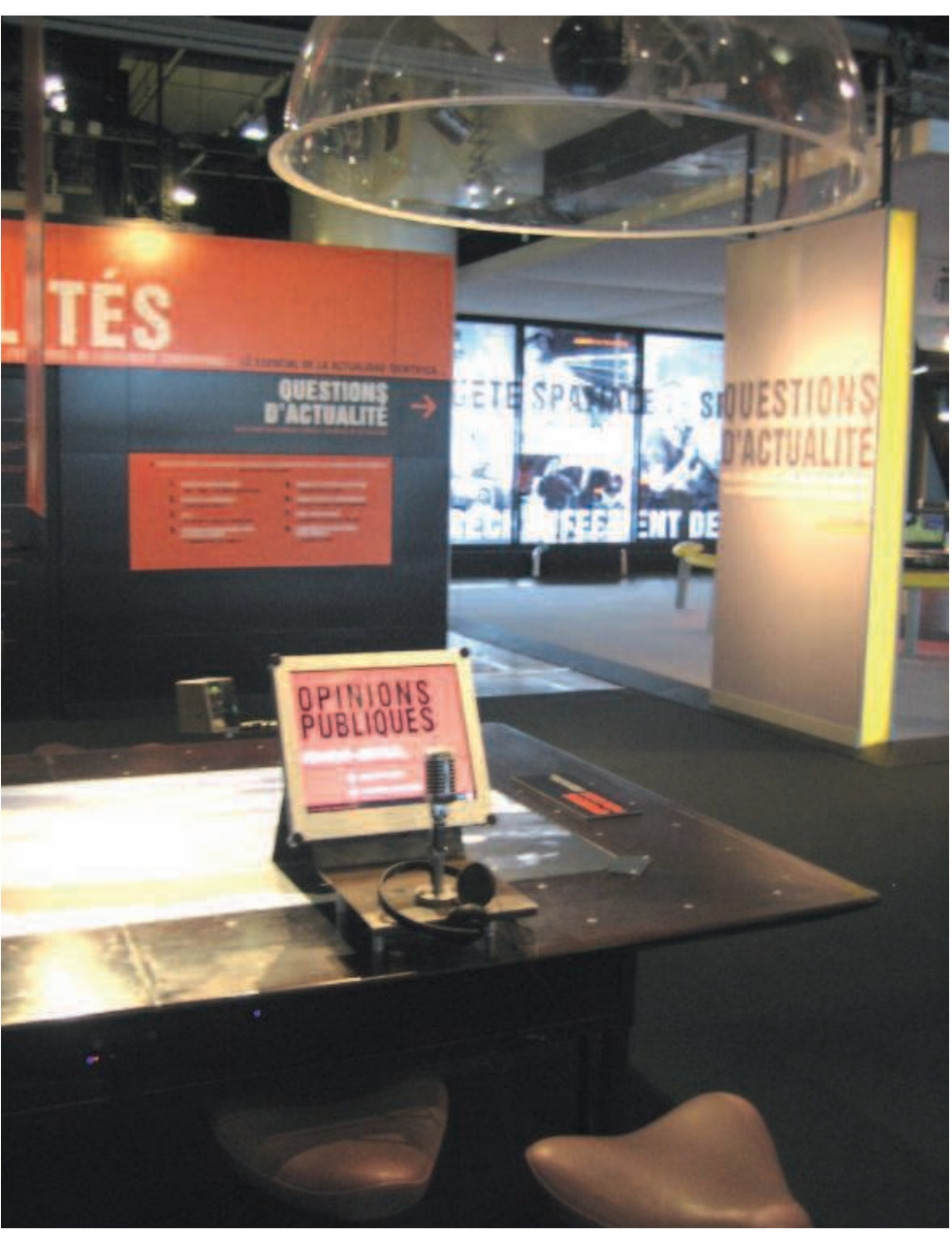

"Opinions publiques», un dispositif vidéo d'expression des visiteurs dans l'exposition Science Actualités mieux perçu l'espace des possibilités de médiation intégrant, outre les technologies disponibles, les expériences de mise en œuvre avec des équipes différentes, et les retours réguliers sur les pratiques des visiteurs. À l'inverse, cette méthode nous a permis d'éviter les impasses d'un mode de conception qui aurait été tourné entièrement vers la proposition de potentialités d'usage toujours plus ouvertes plus nombreuses, au risque de diluer les projets de communication.

Dans le même temps, sur la durée, nous nous sommes rendu compte que les technologies et leur mise en œuvre ne modifiaient pas seulement la médiation des contenus scientifiques, mais pouvaient également intervenir à l'échelle plus large de la communication avec le musée. Dans un contexte où les musées sont tentés de transformer leur public en ensemble de clients ou usagers dont il faudrait stimuler la consommation et obtenir la fidélisation, l'idée est ici de respecter l'attachement et la confiance que les visiteurs témoignent aux institutions scientifiques et culturelles, et de développer les liens avec eux au sein de l'espace élargi mais maîtrisé de ces institutions ${ }^{(4)}$.

\section{Les études d'usage}

En 1989, le département s’adresse à la cellule Évaluation de la direction des Expositions pour analyser les usages d'une sélection de scénarios interactifs. Les concepteurs multimédia s'intéressent aux études d'évaluation non pour vérifier que les objectifs d'apprentissages ou de satisfaction sont atteints, mais pour avoir un retour sur des démarches de conception fondées sur des paris ou des hypothèses fortes. D'une certaine manière, la démarche est déjà celle d'une exploration des possibilités de communication avec les visiteurs, plus que celle de l'optimisation d'un impact sur le visiteur. Cette demande permet à l'équipe d'évaluation d'amplifier très rapidement un type d'analyse qui va s'avérer très féconde : éviter de perdre son temps à tenter d'objectiver et de mesurer une performance, et se consacrer à l'analyse de ce qui fait sens du point de vue des visiteurs, quels que soient la forme ou le moment de la pratique auquel on s'intéresse : l'arrivée dans la Cité des Sciences et de l'Industrie, la visite d'exposition, l'usage d'un élément d'exposition... Pour cela on abandonne délibérément l'idée d'une position de neutralité très externe de l'évaluation, pour entrer également dans la compréhension de ce qui fait sens pour les concepteurs. C'est ce parti pris qui permet de mettre en évidence la puissance de la communication, dans l'attention portée à l'Autre : au visiteur lorsqu'on est concepteur, à l'énonciateur lorsqu'on est visiteur. 
savoir ou «Opinion publique » dans l'exposition dossier Science Actualités donnent la parole aux visiteurs mais dans un cadre où est assumée l'autorité institutionnelle et sa responsabilité ${ }^{(8)}$. Une partie des projets intègre dans son principe même cette sensibilité des visiteurs à l'espace de communication dans lequel ils se trouvent.

Le capital d'études et d'expériences peut nourrir la réflexion sur l'interactivité, et symétriquement la manière dont la recherche sur les publics peut voir certains de ses résultats être continuellement réinvestis dans l'innovation détail du dispositif.

\section{Visite+}

En l'an 2000, la direction générale demande au département Interactivité et Multimédia de concevoir ce qui va devenir Visite+. Dans l'énoncé de la commande figurent les aspects suivants : « Le guidage » (recueil de profil et d'attentes du visiteur, orientation et suivi dans l'offre de la cité, mémorisation de l'expérience de visite, évaluation et valorisation, marketing et fidélisation des visiteurs), « La muséologie » (personnalisation du parcours de l'exposition, proposition de nouvelles interactions), « L'offre de ressources » (accès à des contenus adaptés à chaque visiteur). Nous avons interprété cette commande pour prendre en compte, d'emblée, le fait que les visiteurs de la Cité des Sciences n'ont pas du tout la même conception de la personnalisation que celle qui inspire les démarches marketing : personnaliser, ce n'est pas adapter des offres à ceux à qui on s'adresse, mais intensifier le rapport de communication et rendre plus perceptibles les intentions et les engagements de ceux qui proposent.

L'attente de la Cité s'orientait fortement vers la mise à disposition des visiteurs d'un outil de communication mobile communiquant (PDA) déjà présent dans le commerce mais inadapté à l'époque à l'usage dans le musée. Au plan technique, nous ne pouvions pas nous reposer sur les systèmes existants, ni être en attente de technologies émergentes pour réaliser le projet d'un dispositif atteignant les objectifs souhaités (guidage dans l'exposition, mémorisation de l'expérience de visite, et offre de ressources après la visite), car nous connaissions les délais entre l'annonce d'un nouvel outil et sa mise en œuvre dans un contexte quasi industriel. Plutôt que de réaliser un projet dont l'aboutissement aurait été aléatoire et qui aurait été dépassé dès sa mise en œuvre, nous avons choisi d'utiliser pragmatiquement ce que les technologies éprouvées avaient de meilleur en concevant un développement évolutif qui pourrait s'articuler sans effort

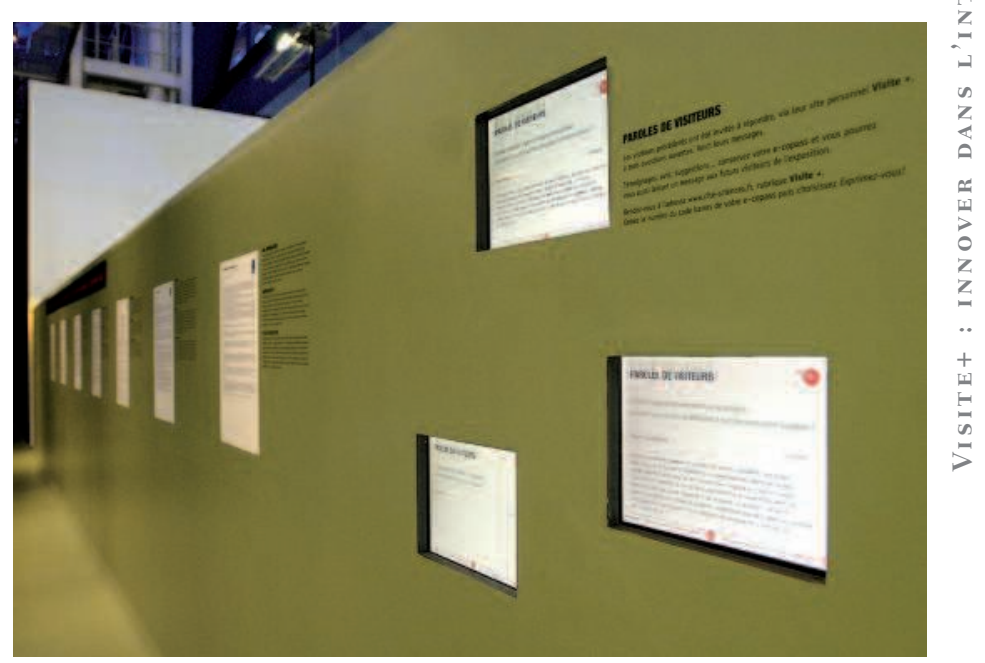

Écrans restituant les avis des visiteurs exprimés à domicile sur leurs Cyber-carnets dans l'exposition Changer d'ère

(c) J.P. Attal

aux technologies émergentes. Il s'agissait donc d'amplifier et d'organiser la démarche détricotage technologique, et de penser d'emblée un dispositif ouvert aux évolutions constantes dans des contextes eux-mêmes changeants. Ainsi, Visite+ s'est appuyé sur des principes issus des technologies numériques qui permettent : la personnalisation, l'interactivité, le suivi de la visite in situ, la poursuite de la visite sur Internet, la visite augmentée par l'utilisation d'outils de médiation mobiles.

En personnalisant la visite, et en prolongeant l'exposition dans l'espace virtuel d'Internet, Visite+ permet la conception de scénarios d'expositions inédits en réalisant un élargissement spatial et temporel de la relation à l'exposition. Il s'agit également de créer et d'entretenir les conditions d'une relation à long terme entre le visiteur et l'institution. Cette démarche vient en rupture de pratiques plus classiques consistant à développer des applications pour une utilisation unique (un site Internet, une exposition). Le développement « incrémental » permet, lui, de capitaliser les services réalisés pour les besoin des expositions. Ce qui a été développé pour une exposition devient disponible pour les autres, et on constitue donc une banque de services potentiellement disponibles aux autres expositions. Dans ce processus, les offres ne sont pas figées. Ainsi La lettre $d u$ visiteur a disparu en 2005.

Au plan de la communication, la connaissance des publics a permis de faire des choix fondateurs : dans le contexte d'une extrême sensibilité des publics, à 


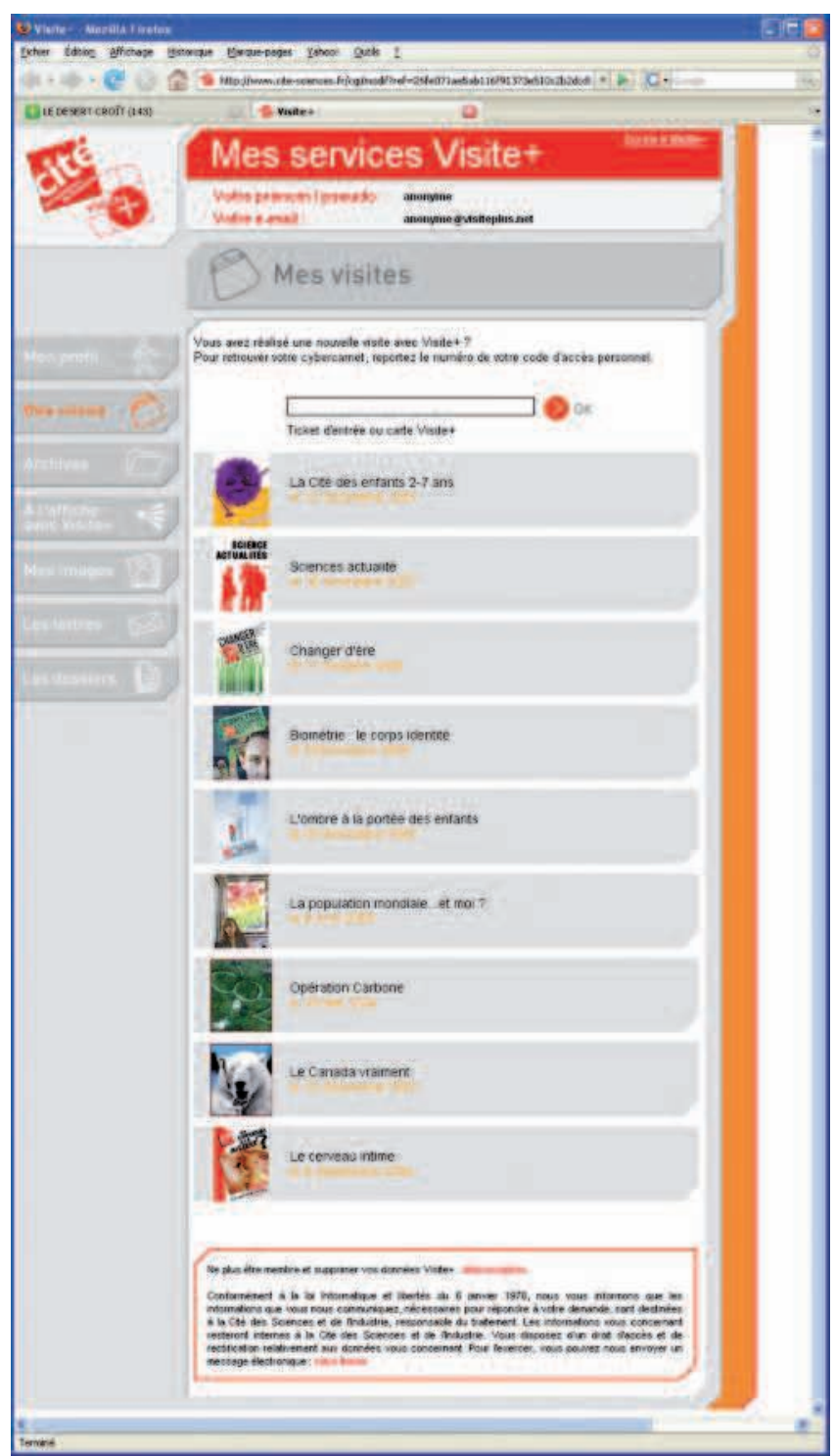

Le portail du visiteur membre de Visite+ avec le rappel de ses visites et l'accès aux autres offres

la différence entre espaces (espace Internet et espace muséal, espace marchand et espace institutionnel) il s'agissait de mettre en avant de manière très claire les choix institutionnels pour respecter les attentes des publics à cet égard : partir de la visite de l'exposition, assumer le fait qu'il ne s'agit pas d'un service dérivé proposé par un opérateur payant à certaines fractions du public, donner des informations au visiteur et non pas capter des informations sur lui-même, renforcer la cohérence de démarches existantes et non les brouiller.

Au plan de la démarche de collaboration associant conception et études, la démarche antérieure a été très amplifiée par rapport à ce que nous avions expérimenté jusqu'ici à l'occasion de réalisations particulières. Nous avons décidé de réfléchir en continu, avec des séminaires fermés et des études menées auprès des visiteurs à chaque mise en accès public d'une version de Visite+. Là encore, cette démarche vient en rupture avec des modes de recherche-développement qui trouvent leur aboutissement dans la mise au point d'un objet technique ou d'une réalisation spécifique.

\section{Description de Visite+}

Il y a plusieurs manières de décrire Visite+. Nous éviterons celle qui développerait la combinaison des technologies déployées, pour privilégier celle qui détaille le parti pris de médiation. Mais à son tour celui-ci s'énonce différemment selon qu'on décrit le lien de Visite+ aux expositions auxquelles il est associé ou selon que l'on observe l'effet sur les pratiques et la relation aux visiteurs. Si l'on part du lien entre Visite+ et l'exposition, le parti pris fondateur est le suivant : dans la mesure où on accorde une importance centrale au lien à l'institution et à la spécificité des pratiques, le dispositif déploie l'ensemble de ses propositions non pas hors visite (sur Internet directement), mais à partir de celle-ci, puis dans son extension sur Internet, et enfin dans la réflexion sur l'articulation à d'autres pratiques culturelles, dans d'autres temporalités. C'est pourquoi les internautes qui s'inscrivent en ligne et n'ont pas visité n'ont pas accès aux mêmes contenus.

\section{Dans l'exposition}

Un identifiant est distribué à chaque visiteur ${ }^{(9)}$. En 2002 dans l'exposition Le cerveau intime, c'est un code barre sur le ticket d'entrée. Lors de la visite, le visiteur signale son passage et peut saisir des éléments le concernant. Il s'agit non d'établir son profil-type, mais de ne garder que des caractéristiques pertinentes, utiles à la présentation du contenu de l'exposition. Ainsi, dans La population mondiale et moi, les éléments multimédia, après avoir enregistré la langue, l'âge et le sexe du visiteur, présentent dans la langue du visiteur les données démographiques rapportées à son cas particulier. À l'inverse dans l'exposition Changer d'ère, les éléments de contenu apportés par les visiteurs sont agrégés pour développer des contenus collectifs (par exemple l'empreinte écologique cumulée ou un questionnaire sur le développement durable).

Avec son identifiant, le visiteur peut signaler son passage, déclencher des éléments multimédia, répondre à certaines questions, participer à des votes, déclarer son intérêt pour une œuvre, enregistrer 


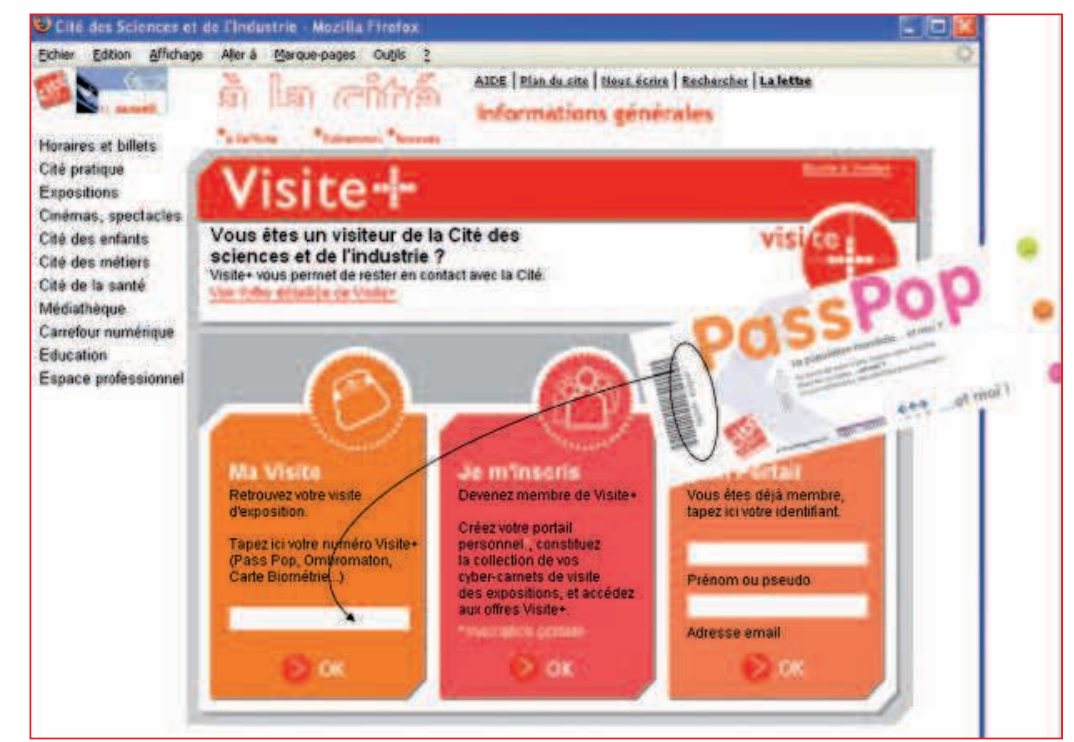

Accès au Cyber-carnet pour la post visite sur Internet

La population mondiale et moi. On évite ainsi, au fil des expérimentations, d'ouvrir toujours plus grand un spectre de potentialités techniques qui serait contradictoire avec cette recherche de cohérence.

\section{La post visite, site mémoire et lieu d'expression pour socialiser l'expérience de visite}

Les études menées actuellement sur les versions successives de Visite+ concernent essentiellement l'usage pendant la visite et l'anticipation des usages du site. Avec La population mondiale et moi, nous avons commencé à interroger certaines personnes chez elles quelques semaines après la visite.

Comme nous l'avons déjà évoqué, Visite+ permet la poursuite, l'enrichissement et le partage de l'expérience de visite sur un site Internet personnel, le Cyber-carnet de visite, accessible à domicile ou en classe aux visiteurs qui se sont enregistrés dans l'exposition. Il s'avère au fil des études réalisées dans Le cervean intime et La population mondiale et moi sur la manière dont les visiteurs anticipent ce qu'ils pourront faire après la visite, que Visite+ permet d'amplifier les logiques très différentes qui sous-tendent les pratiques : les enseignants se représentent les possibilités de travail sur les contenus de l'exposition après la visite de celle-ci lors de visites studieuses, certains visiteurs à l'inverse s'imaginent revivre des sensations et des impressions lorsqu'ils ont vécu la visite comme une expérience. Dans de nombreux cas, on évoque les possibilités de discussion et de partage avec autrui, en premier lieu les proches qui n'ont pas visité, mais aussi « les gens du musée » à qui sont effectivement envoyés des messages :

- Mémoire de l'exposition : le visiteur peut retrouver l'ensemble des contenus présentés dans l'exposition que ce soient les textes, les vidéos, les photographies d'objets... le tout assorti des contenus complémentaires qui ont nourri toute la phase de conception de l'exposition, ainsi que des liens vers des sites présentant des contenus en relation avec les thématiques de l'exposition : musées, partenaires laboratoires, presse...

- Mémoire de l'expérience de visite : le visiteur retrouve là tous les éléments personnels liés à son expérience de visite. D’abord son parcours réellement effectué, comme repère rappelant le déroulement de sa visite. Les éléments d'exposition consultés lui sont indiqués, lui permettant d'en retrouver les contenus, et les autres, non consultés, sont à découvrir virtuellement. Le visiteur trouve également les productions réalisées pendant la visite : prise de position dans une vidéo enregistrée, photographie prise dans le contexte de la visite... Il peut également poursuivre une activité (un jeu par exemple) commencé dans l'exposition.

- Lieu d'expression : on sait à quel point les sites institutionnels se prêtent difficilement au développement de forums et espaces de discussion. Cependant, forts des précédentes expériences Livre d'or et La tribune des visiteurs, nous gardons l'objectif de faire en sorte que Visite+ puisse être l'espace d'expression pour la communauté virtuelle des visiteurs d'exposition (témoignage d'expériences, commentaires critiques...). Il s'agit d'offrir un cadre éditorial et vivant à une " expophilie ", une culture des expositions qui n'a guère d'espaces d'expression actuellement. Les interventions des visiteurs, après modération, sont accessibles soit dans l'espace partagé de l'espace virtuel des visiteurs (accessible par les Cyber-carnets), soit sur Internet ou encore présentées dans l'exposition elle-même. Ainsi via Visite+, les visiteurs sont amenés à faire vivre et évoluer l'exposition.

- Socialiser l'expérience de visite : le visiteur peut présenter sa visite à ses amis ou à sa famille la partager, rejouer avec eux aux activités proposées, ou revoir les films et les présentations. Il peut envoyer son Cyber-carnet à des parents par mél ou le placer sur son blog. L'enseignant peut l'utiliser pour préparer et poursuivre la visite avec ses élèves.

- Sur le Web, entretenir la relation visiteur-institution : chaque visiteur a la possibilité de devenir membre de Visite+, dès lors, il accède à un portail 
privé où il collectionne ses Cyber-carnets des expositions visitées. Il peut aussi télécharger nombre de dossiers bibliographiques des expositions et potentiellement accéder à toute offre personnalisée que l'institution souhaite lui adresser.

Cette proposition de collection des visites est un des développements que nous souhaitons mutualiser avec les institutions partenaires afin d'offrir à chaque visiteur la collection de ses visites culturelles toutes institutions confondues

\section{L'extension de Visite+, la diffusion de la licence et la création d'une plate-forme musées/recherche}

L'accès à Visite+, jusque-là limité à la seule Cité des Sciences et de l'Industrie, est désormais ouvert à toute institution publique sous forme de licence gratuite. Plusieurs institutions muséales et sites culturels français et étrangers, ainsi que des laboratoires s'intéressent à la mise en œuvre de ce système. Cependant, Visite+ par nature évolue en permanence en fonction des applications et des usages qu'en font les visiteurs, et des évolutions technologiques. C'est la démarche et l'ensemble des propositions auxquelles elle aboutit qui constitue le " produit ». C'est pourquoi cette mise en accès n'est pas suffisante. Visite+ implique un mode de conception spécifique et donc une innovation institutionnelle: une plate-forme de mise en commun non pas d'outils et de ressources, mais d'une démarche partagée.

Cette proposition est justifiée à plusieurs titres, par rapport à l'intérêt des publics et des institutions, par rapport aux exigences de développement technologique et par rapport aux enjeux de cumul et de partage de savoirs et d'expertise entre institutions muséales et recherche mais aussi pour proposer des usages nouveaux comme, par exemple, associer Visite+ aux audioguides et utiliser simplement les données recueillies par l'audioguide durant la visite pour réaliser simplement des post visites permettant de retrouver le parcours et les commentaires associés à la visite

- Pour l'intérêt des publics et celui des institutions : la visite des musées et centres de culture scientifique s'inscrit dans des pratiques culturelles dont le sens n'est évidemment pas limité par les frontières de tel ou tel établissement et ce sont les publics qui créent le réseau culturel de leurs propres pratiques. Il est dans l'intérêt des établissements de tirer parti de cette caractéristique désormais bien établie, et donner aux visiteurs un support matériel repérable à cette mise en réseau. Par exemple, Visite+ pourrait permettre aux publics de récapituler et collectionner sur un même dispositif leurs visites, dans différents lieux et tout au long de leur « carrière » de visiteurs. Symétriquement l'ensemble des institutions partenaires pourraient travailler non pas à partir de stratégies d'élargissement de leur propre public, mais plutôt en tenant compte du fait que les publics, exactement comme les professionnels, circulent entre lieux culturels, cherchent le contraste ou bien à l'inverse les proximités.

- Pour le développement de la création en matière de médiation technologique: Visite+ s'incarne dans une diversité de supports, qui varie en fonction des contextes muséographiques, de l'évolution des moyens, et des idées de médiation qui émergeront des besoins propres aux établissements, ou de l'observation des usages. La plate-forme doit permettre à chacune des institutions partenaires de faire évoluer le dispositif, en fonction de ses besoins propres, en acceptant que ces évolutions se fassent au bénéfice des autres institutions. De plus, mettre en commun les développements dans un cadre collectif, c'est en partager les coûts et permettre à chacun de bénéficier des évolutions réalisées dans le cadre de la plateforme.

- Pour le cumul d'expertise et la mise en commun des résultats de recherche : la proposition vise aussi à mutualiser et partager la réflexion, les études et recherches menées sur les usages de Visite+. À tout

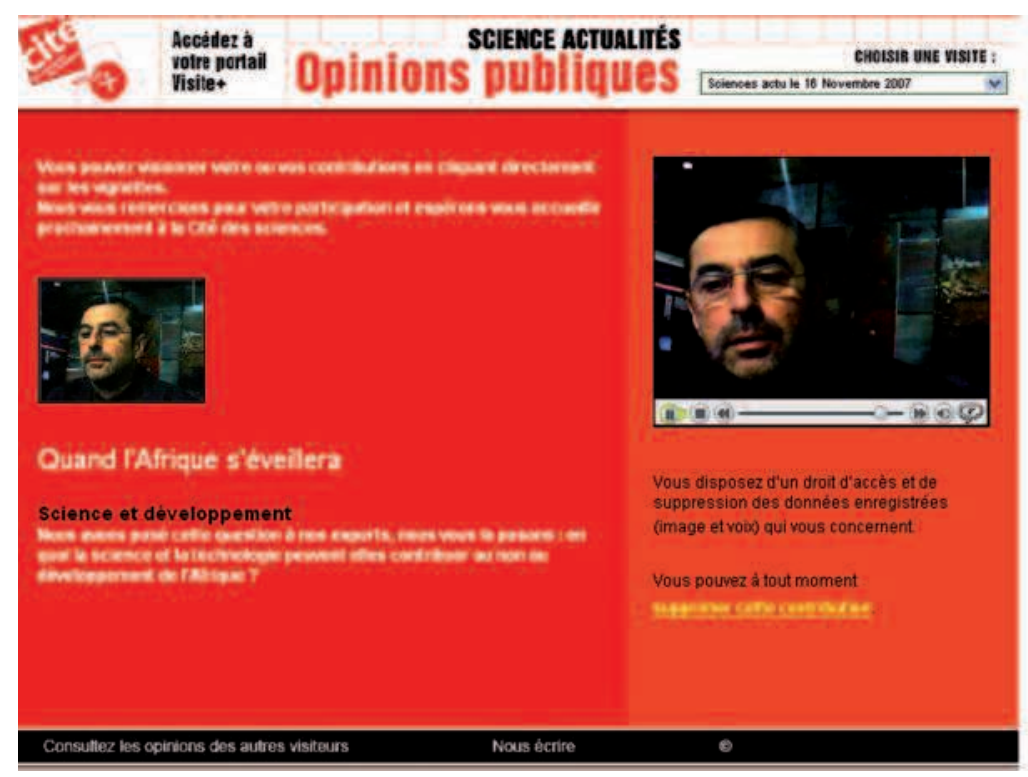

Sur Internet, le Cyber-carnet de l'exposition Science Actualités permet de revoir la vidéo enregistrée sur l'élément "Opinion Publique », et permet aussi de la supprimer à distance. 
moment, les membres de la plate-forme peuvent se constituer en groupe de réflexion avec les chercheurs en sciences sociales associés, pour soumettre des observations, des questions, des propositions.

Dans un contexte où l'étude et l'expertise menées par des sociétés d'étude sont souvent coûteuses pour les institutions de service public, ponctuelles dans leur portée, confidentielles dans leur diffusion, le dispositif proposé fait fonctionner à plein le partage des savoirs entre établissements publics, et permet de développer une recherche développement collective, et continue.

\section{Notes}

(1) Voir Duconseille, P. Le Compagnon : un outil unique d'interaction et de personnalisation de l'Exposition Imaginer le Canada et Botbol, D. Les nouveaux médias à la Cité des Sciences et de l'Industrie, Actes de l'ICHIM, Paris : École du Louvre, septembre 2003.

(2) Voir notamment les études suivantes : Rinçon, L. et Le Marec, J. Évaluation qualitative des pratiques de l'exposition « La population mondiale...et moi ». Étude réalisée pour le département Évaluation et Prospective CSI, novembre 2005 ; Filippini-Fantoni, S. et Le Marec, J. Personnalisation de l'offre aux visiteurs pour l'aide à la conception du «Navigateur ». ENS-LSH, CSI, 2004 ; Gauzins, E. et Le Marec, J. Réactions des visiteurs à Visite+ Premier bilan. ENS-LSH, Association Ad Hoc, CSI, 2003 ; Deshayes, S. et Le Marec, J. Le projet « Navigateur » à la Cité des Sciences et de l'Industrie, compte-rendu d'expertise. ENSLSH, Association Publics en Perspective, 2002.

(3) Voir Goldstein, B., Le Marec, J., Pouts-Lajus, S. et Topalian, R. Interactifs : fonctions et usages dans les musées. Paris : Direction des Musées de France, 1996.

(4) Dans une recherche effectuée pour le développement de l'usage des téléphones mobiles pour la visite culturelle, Sophie Deshayes (laboratoire C2SO, ENS Lettres Sciences humaines) a montré la différence remarquable de la valeur attribuée à une proposition (et du consentement à payer pour elle) selon que celle-ci apparaît comme émanant de l'opérateur téléphonique, qui inspire méfiance et désenchantement, ou bien du musée.

(5) Voir Le Marec, J. Les musées et bibliothèques comme espaces culturels de formation, Savoirs, n¹1, 2006.

(6) Par contre, cette opposition commence à structurer des positionnements professionnels autour de la médiation et de la muséographie dans les années 1990, ce qui suscite un malentendu important : une minoration systématique de l'attachement des publics au lien à l'institution et au goût pour les savoirs et une surinterprétation du goût pour les technologies de la communication.

(7) Deshayes, S. Audioguides et musées, la Lettre de l'OCIM, n ${ }^{\circ}$, janvier-février 2002
(8) Le Marec, J. et Topalian, R. Énonciation plurielle et publication de la parole du public en contexte muséal : le cas de « La tribune des visiteurs ", Communication et Langages, n¹38, 2003.

(9) L'identifiant peut prendre différentes formes : un bracelet, un ticket avec un code barre, un « tag » RFID, un assistant personnel (PDA) relié ou non à wifi, ou encore un audio guide. Les mesures biométriques sont à proscrire car les technologies sont trop suspectes aux yeux des visiteurs. (10) Les éléments Visite+ sont les moyens techniques et logiciels, fixes ou embarqués, intégrés à la visite pour mettre en œuvre le scénario Visite+. Ces éléments peuvent être apparents ou transparent dans l'exposition. Une exposition Visite+ est constituée de bornes fixes et d'identifiants portés par les visiteurs.

\section{Bibliographie}

Dédale Les institutions culturelles dans leur rôle de nouveaux environnements d'apprentissage. Commission européenne, Direction générale Éducation et Culture, Unité Multimédia, 2004 (www.dedale.info/etude_elearning).

Guichard, E. (dir.) Comprendre les usages de l'Internet. Paris : Éditions ENS, 2001.

Jeanneret, Y. Y a-t-il (vraiment) des technologies de l'information? Lille : Presses du Septentrion, 2000.

Le Marec, J. Les études d'usage des multimédias en milieu culturel : une évolution des questions, Culture et recherche, $\mathrm{n}^{\circ} 102,2004$ (www.culture.gouv.fr/culture/editions/r-cr/cr102.pdf).

Le Marec, J et Topalian, R Le rôle des technologies dans les relations entre les institutions et les publics : peut-on (vraiment) innover en matière de communication ?, Actes de l'ICHIM, 2003 (www.archimuse.com/publishing/ichim03/056C.pdf).

Schiele, B. Le musée des sciences : montée du modèle communicationnel et recomposition du champ muséal. Paris : l'Harmattan, 2001.

Topalian, R. Visite+ : personnalisation de la visite et site mémoire des visites culturelles, Culture et recherche, ${ }^{\circ} 112,2007$ (www.culture.gouv.fr/ culture/editions/r-cr/crl 12.pdf).

Topalian, R. Cultural Visit Memory : the Visite+ System Personalization and Cultural Visit Tracking Site, Museum and the Web, 2005, (www.archimuse.com/mw2005/papers/topalian/topalian.html).

Trent, J. et Bearman, D. (dir.) Museums and the web, Actes de l'ICHIM, 2007, Toronto (www.archimuse.com/publishing/mw_2007_TOC.html). 